\title{
Discovering Colocation Patterns from Spatial Data Sets: A General Approach
}

\author{
Yan Huang, Member, IEEE Computer Society, Shashi Shekhar, Fellow, IEEE Computer Society, and \\ Hui Xiong, Student Member, IEEE Computer Society
}

\begin{abstract}
Given a collection of Boolean spatial features, the colocation pattern discovery process finds the subsets of features frequently located together. For example, the analysis of an ecology data set may reveal symbiotic species. The spatial colocation rule problem is different from the association rule problem since there is no natural notion of transactions in spatial data sets which are embedded in continuous geographic space. In this paper, we provide a transaction-free approach to mine colocation patterns by using the concept of proximity neighborhood. A new interest measure, a participation index, is also proposed for spatial colocation patterns. The participation index is used as the measure of prevalence of a colocation for two reasons. First, this measure is closely related to the cross- $K$ function, which is often used as a statistical measure of interaction among pairs of spatial features. Second, it also possesses an antimonotone property which can be exploited for computational efficiency. Furthermore, we design an algorithm to discover colocation patterns. This algorithm includes a novel multiresolution pruning technique. Finally, experimental results are provided to show the strength of the algorithm and design decisions related to performance tuning.
\end{abstract}

Index Terms-Colocation patterns, spatial association rules, participation index.

\section{INTRODUCTION}

C OLOCATION patterns represent subsets of Boolean spatial features whose instances are often located in close geographic proximity. Fig. 1 shows a data set consisting of instances of several Boolean spatial features, each represented by a distinct shape. A careful review reveals two colocation patterns, i.e., $\left\{{ }^{\prime}+,{ }^{\prime} x^{\prime}\right\}$ and $\left\{{ }^{\prime} 0,{ }^{\prime}{ }^{\prime *}\right\}$. Real-world examples of colocation patterns include symbiotic species, e.g., the Nile Crocodile and Egyptian Plover in ecology. Boolean spatial features describe the presence or absence of geographic object types at different locations in a twodimensional or three-dimensional metric space, such as the surface of the earth. Examples of Boolean spatial features include plant species, animal species, road types, cancers, crime, and business types.

Colocation rules are models to infer the presence of spatial features in the neighborhood of instances of other spatial features. For example, "Nile Crocodiles $\rightarrow$ Egyptian Plover" predicts the presence of Egyptian Plover birds in areas with Nile Crocodiles.

We formalize the colocation rule mining problem as follows: Given 1) a set $T$ of $K$ spatial feature types $T=$ $\left\{f_{1}, f_{2}, \ldots, f_{K}\right\}$ and their instances $I=\left\{i_{1}, i_{2}, \ldots, i_{N}\right\}$, each $i_{i} \in \mathrm{I}$ is a vector < instance-id, spatial feature type, location $>$, where location $\in$ spatial framework $S$ and 2) a neighbor relation $\mathcal{R}$ over instances in $I$, efficiently

- Y. Huang is with the Department of Computer Science and Engineering, University of North Texas, PO Box 311366, Denton, TX 76203. E-mail: huangyan@unt.edu.

- S. Shekhar and H. Xiong are with the Department of Computer Science and Engineering, University of Minnesota, 200 Union Street SE, Minneapolis, MN 55455. E-mail: \{Shekhar, huix\}@cs.umn.edu.

Manuscript received 26 Aug. 2002; revised 19 June 2003; accepted 27 Apr. 2004.

For information on obtaining reprints of this article, please send e-mail to: tkde@computer.org, and reference IEEECS Log Number 117191. find all the colocated spatial features in the form of subsets of features or rules.

\subsection{Related Work}

Approaches to discovering colocation rules in the literature can be categorized into two classes, namely, spatial statistics and data mining approaches. Spatial statistics-based approaches use measures of spatial correlation to characterize the relationship between different types of spatial features. Measures of spatial correlation include the cross- $K$ function with Monte Carlo simulation [6], mean nearest-neighbor distance, and spatial regression models [5]. Computing spatial correlation measures for all possible colocation patterns can be computationally expensive due to the exponential number of candidate subsets given a large collection of spatial Boolean features.

Data mining approaches can be further divided into a clustering-based map overlay approach and association rule-based approaches. A clustering-based map overlay approach [9], [8] treats every spatial attribute as a map layer and considers spatial clusters (regions) of point-data in each layer as candidates for mining associations. Given $X$ and $Y$ as sets of layers, a clustered spatial association rule is defined as $X \Rightarrow Y(C S, C C \%)$, for $X \cap Y=\emptyset$, where CS is the clustered support, defined as the ratio of the area of the cluster (region) that satisfies both $X$ and $Y$ to the total area of the study region $S$, and $C C \%$ is the clustered confidence, which can be interpreted as $C C \%$ of areas of clusters (regions) of $X$ intersect with areas of clusters (regions) of $Y$.

Association rule-based approaches can be divided into transaction-based approaches and distance-based approaches. Transaction-based approaches focus on defining transactions over space so that an Apriori-like algorithm [2] can be used. Transactions over space can be defined by a reference-feature centric model [12]. Under this model, transactions are created around instances of one userspecified spatial feature. The association rules are derived using the Apriori [2] algorithm. The rules found are all 


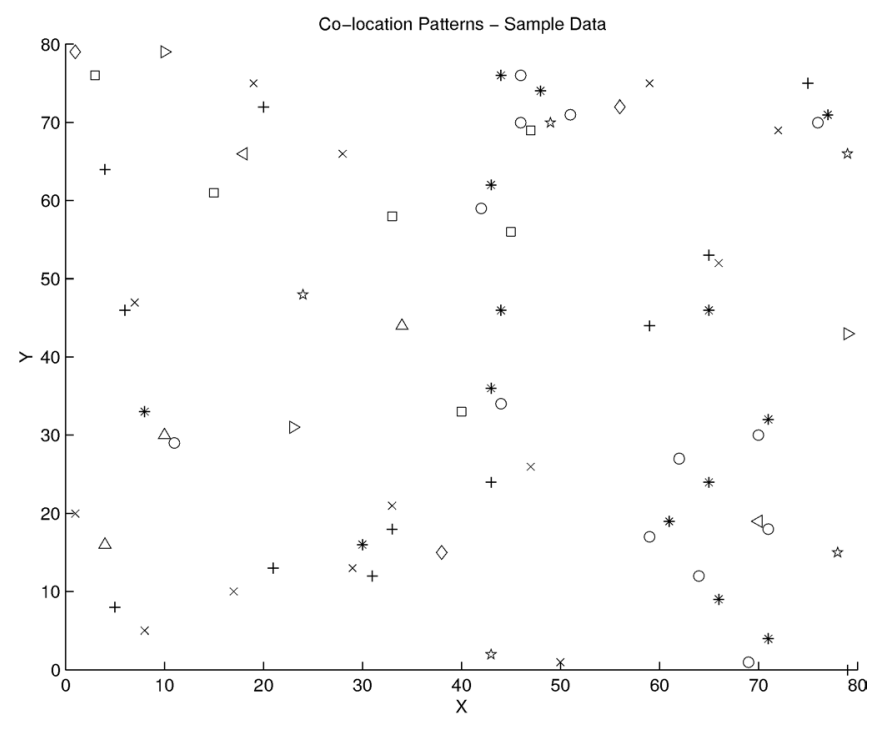

Fig. 1. Colocation patterns illustration.

related to the reference feature. However, generalizing this paradigm to the case where no reference feature is specified is nontrivial. Also, defining transactions around locations of instances of all features may yield duplicate counts for many candidate associations.

A distance-based approach was proposed concurrently by Morimoto [15] and Shekhar and Huang [19]. Morimoto defined distance-based patterns called k-neighboring class sets. In his work, the number of instances for each pattern is used as the prevalence measure, which does not possess an antimonotone property by nature. However, Morimoto used a nonoverlapping instance constraint to get the antimonotone property for this measure. In contrast, we developed an event centric model, which does away with the nonoverlapping instance constraint. We also defined a new prevalence measure called the participation index. This measure possesses the desirable antimonotone property. A more detailed comparison of these two works is presented in Section 6.

\subsection{Contributions}

This paper extends our work [19] on the event centric model and makes the following contributions. First, we refine the definition of distance-based spatial colocation patterns by providing an interest measure called the participation index. This measure not only possesses a desirable antimonotone property for efficiently identifying colocation patterns, but also allows for formalizing the correctness and completeness of the proposed algorithm. Furthermore, we show the relationship between the participation index and a spatial statistics interest measure, the cross- $K$ function. More specifically, we show that the participation index is an upper-bound of the cross-K function. Second, we provide an algorithm to discover colocation patterns from spatial point data sets. This algorithm includes a novel multiresolution filter, which exploits the spatial autocorrelation property of spatial data to effectively reduce the search space. An experimental evaluation on both synthetic and real-world NASA climate data sets is provided to compare alternative choices for key design decisions.

\subsection{Outline and Scope}

Section 2 describes our approach for modeling colocation patterns. Section 3 proposes a family of algorithms to mine colocation patterns. We show the relationship between the participation index and an estimator of the cross- $\mathrm{K}$ function and provide an analysis of the algorithms in the area of correctness, completeness, and computational efficiency in Section 4. In Section 5, we present the experimental setup and results. Section 6 provides a comparison between our work and the work by Morimoto [15]. Finally, in Section 7, we present the conclusion and future work.

This paper does not address issues related to the edge effects or the choice of the neighborhood size and interest measure thresholds. Quantitative association, e.g., $(A, A)$ and quantitative association rules, e.g., $(A \Rightarrow A)$, are beyond the scope of this paper.

\section{Our Approach for Modeling Colocation PATTERNS}

This section defines the event centric model, our approach to modeling colocation patterns. We use Fig. 2 as an example spatial data set to illustrate the model. In the figure, each instance is uniquely identified by $T . i$, where $T$ is the spatial feature type and $i$ is the unique id inside each spatial feature type. For example, B.2 represents the instance 2 of spatial feature $B$. Two instances are connected by edges if they have a spatial neighbor relationship.

A colocation is a subset of Boolean spatial features. A colocation rule is of the form: $c_{1} \Rightarrow c_{2}(p, c p)$, where $c_{1}$ and $c_{2}$ are colocations, $c_{1} \bigcap c_{2}=\emptyset, p$ is a number representing the prevalence measure, and $c p$ is a number measuring conditional probability.

An important concept in the event centric model is proximity neighborhood. Given a reflexive and symmetric neighbor relation $\mathcal{R}$ over a set $S$ of instances, a $\mathcal{R}$-proximity neighborhood is a set $I \subset S$ of instances that form a clique [4] under the relation $\mathcal{R}$. The definition of neighbor relation $\mathcal{R}$ is an input and should be based on the semantics of the application domains. The neighbor relation $\mathcal{R}$ may be defined using spatial relationships (e.g., connected, adjacent [1]), metric relationships (e.g., Euclidean distance [15]), or a combination (e.g., shortest-path distance in a graph such as a roadmap). The $\mathcal{R}$-proximity neighborhood concept is different from the neighborhood concept in topology [14] since some supersets of a $\mathcal{R}$-proximity neighborhood may not qualify to be $\mathcal{R}$-proximity neighborhoods.

Two $\mathcal{R}$-proximity neighborhoods $I_{1}$ and $I_{2}$ are $\mathcal{R}$-reachable to each other if $I_{1} \cup I_{2}$ is a $\mathcal{R}$-proximity neighborhood. A $\mathcal{R}$-proximity neighborhood $I$ is a row instance (denoted by row_instance $(c)$ ) of a colocation $c$ if $I$ contains instances of all the features in $c$ and no proper subset of $I$ does so. For example, $\{A .3, B .4, C .1\}$ is a row instance of colocation $\{A, B, C\}$ in the spatial data set shown in Fig. 2. But, $\{A .2, A .3, B .4, C .1\}$ is not a row instance of colocation $\{A, B, C\}$ because its proper subset $\{A .3, B .4, C .1\}$ contains instances of all features in $\{A, B, C\}$. In another example, $\{A .2, A .4\}$ is not a row instance of colocation $\{A\}$ because its proper subset $\{A .2\}$ (or $\{A .4\}$ ) contains instances of all the features in $\{A\}$. The table instance, table_instance $(c)$, of a colocation $c$ is the collection of all row instances of $c$. In Fig. 2, 


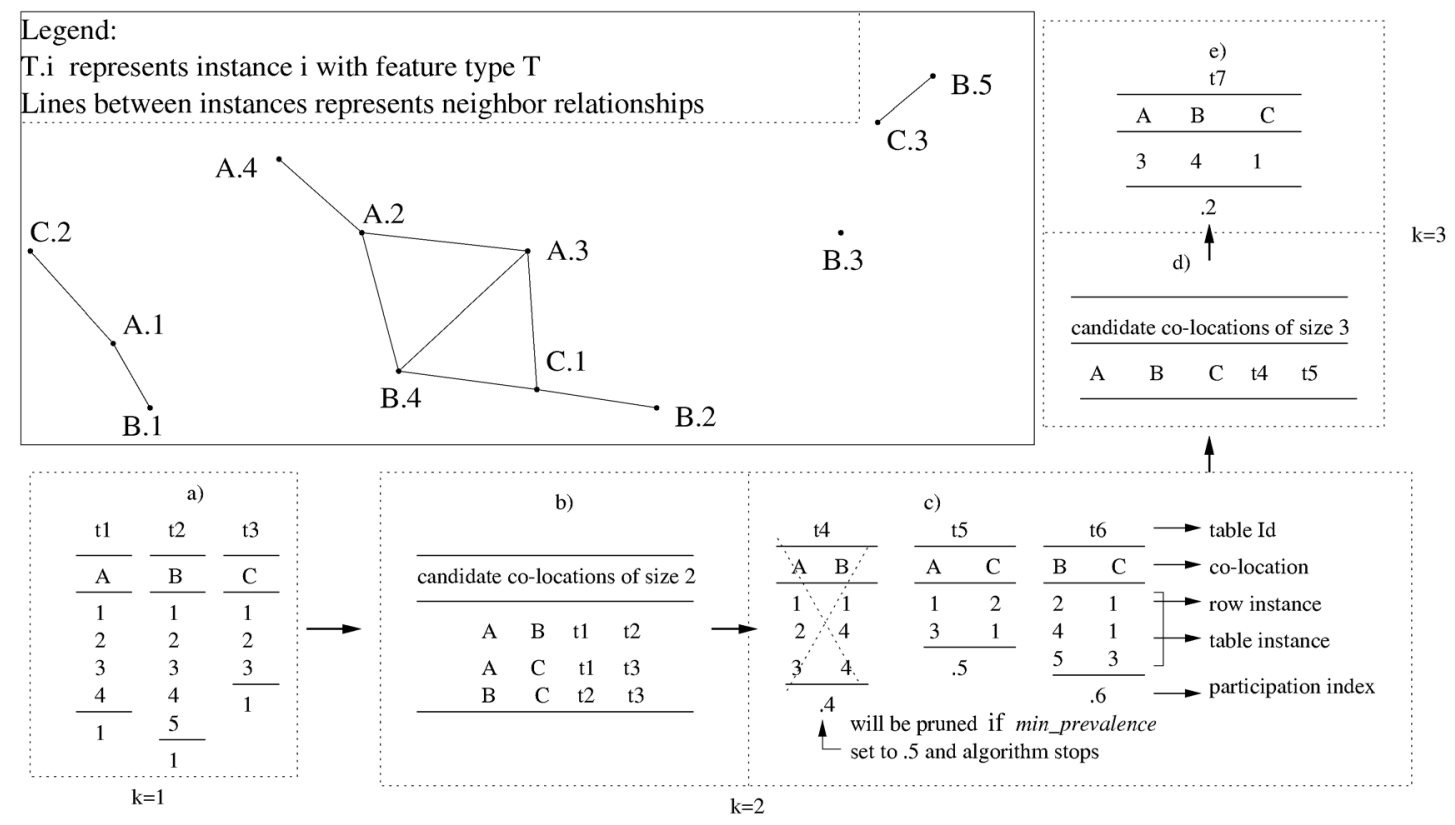

Fig. 2. Spatial data set to illustrate the event centric model.

$\mathrm{t} 1, \mathrm{t} 2, \mathrm{t} 3, \mathrm{t} 4, \mathrm{t} 5, \mathrm{t} 6$, and $\mathrm{t} 7$ represent table instances. For instance, $\mathrm{t} 5=\{\{$ A. 1, C. 2$\},\{$ A. .3, C. 1$\}\}$ is a table instance of the colocation $\{\mathrm{A}, \mathrm{C}\}$.

The participation ratio $\operatorname{pr}\left(c, f_{i}\right)$ for feature type $f_{i}$ in a size-k colocation $c=\left\{f_{1}, \ldots, f_{k}\right\}$ is the fraction of instances of feature $f_{i} \mathcal{R}$-reachable to some row instance of colocation $c-\left\{f_{i}\right\}$. The participation index $p i(c)$ of a colocation $c=$ $\left\{f_{1}, \ldots, f_{k}\right\}$ is $\min _{i=1}^{k}\left\{\operatorname{pr}\left(c, f_{i}\right)\right\}$. The participation index is used as the measure of prevalence of a colocation for two reasons. First, the participation index is closely related to the cross- $K$ function [16], [17] which is often used as a statistical measure of interaction among pairs of spatial features. Second, it also possesses an antimonotone property which can be exploited for computational efficiency. The participation ratio can be computed as

$$
\frac{\pi_{f_{i}}(\mid \text { table_instance }(c) \mid}{\mid \text { table_instance }\left(f_{i}\right) \mid},
$$

where $\pi$ is the relational projection operation with duplication elimination. For example, in Fig. 2, row instances of colocation $\{A, B\}$ are $\{\{A .1, B .1\},\{A .2, B .4\},\{A .3, B .4\}\}$. Only two $(B .1$ and $B .4$ ) out of five instances of spatial feature $B$ participate in colocation $\{A, B\}$. So, $\operatorname{pr}(\{A, B\}, B)=2 / 5=0.4$. Similarly, $\operatorname{pr}(\{A, B\}, A)$ is 0.75 . The participation index $\operatorname{pi}(\{A, B\})=$ $\min (0.75,0.4)=0.4$.

The conditional probability $c p\left(c_{1} \Rightarrow c_{2}\right)$ of a colocation rule $c_{1} \Rightarrow c_{2}$ is the fraction of row instances of $c_{1} \mathcal{R}$-reachable to some row instance of $c_{2}$. It is computed as

$$
\frac{\mid \pi_{c_{1}}\left(\text { table_instance }\left(\left\{c_{1} \bigcup c_{2}\right\}\right)\right) \mid}{\mid \text { table_instance }\left(\left\{c_{1}\right\}\right) \mid},
$$

where $\pi$ is the relational projection operation with duplication elimination. For example, in the colocation rule $A \Rightarrow C$ in Fig. 2, the conditional probability of this rule is equal to

$$
\frac{\mid \pi_{A}(\text { table_instance }(\{A \cup C\})) \mid}{\mid \text { table_instance }(\{A\})}=\frac{2}{4}=50 \% \text {. }
$$

\section{Colocation Mining Algorithm}

In this section, we introduce a colocation mining algorithm. Note that the prevalence measure used in Fig. 3 is the participation index and that a colocation pattern is prevalent if the values of its participation index is above a user specified threshold.

As shown in Fig. 3, the algorithm takes a set ET of spatial event types, a set $E$ of event instances, user-defined functions representing spatial neighborhood relationships as well as thresholds for interest measures, i.e., prevalence and conditional probability. The algorithm outputs a set of prevalent colocation rules with the values of the interest measures above the user defined thresholds.

The initialization steps (i.e., steps 1 and 2 in Fig. 3) assign starting values to various data structures used in the algorithm. We note that the value of the participation index is 1 for all colocations of size 1 . In other words, all colocations of size 1 are prevalent and there is no need for either the computation of a prevalence measure or prevalence-based filtering. Thus, the set $C_{1}$ of candidate colocations of size 1 as well as the set $P_{1}$ of prevalent colocations of size 1 are initialized to $E T$, the set of event types. The set $T_{1}$ of table instances of size 1 colocation is created by sorting the set $E$ of event instances by event types. If a multiresolution pruning step is desired, the set of events are discretized into coarse level instances. The set $T_{-} C_{1}$ of coarse-level table instances of size 1 colocations is generated by sorting the coarse-level event instances by event types.

The proposed algorithms for mining colocation rules iteratively perform four basic tasks, namely, generation of candidate colocations, generation of table instances of candidate colocations, pruning, and generation of colocation rules. These tasks are carried out inside a loop iterating over the size of the colocations. Iterations start with size 2 since our definition of prevalence measure allows no pruning for colocations of size 1 . 


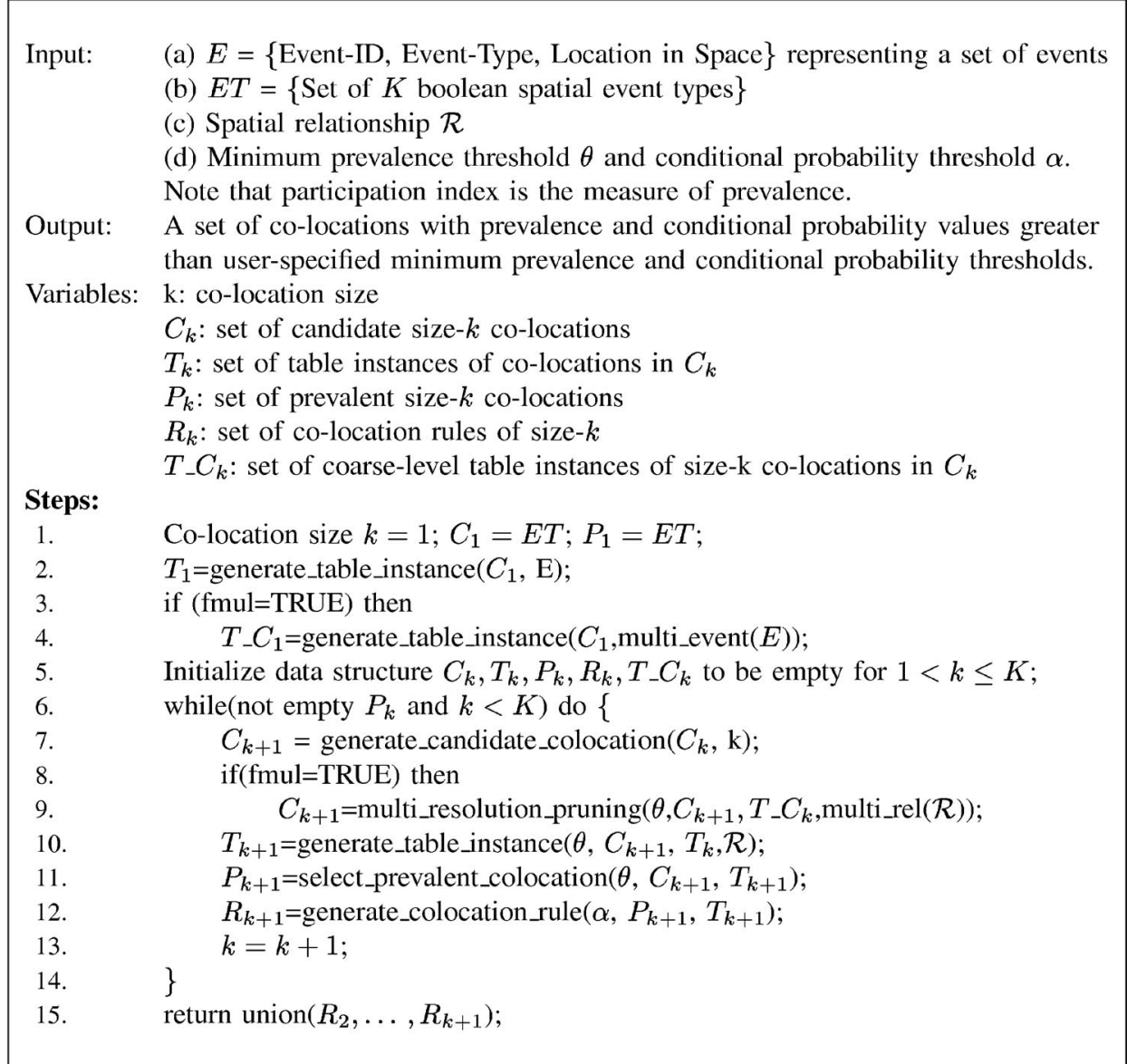

Fig. 3. Overview of the algorithms.

\subsection{Generation of Candidate Colocations}

We could rely on a combinatorial approach and use apriori_gen [2] to generate size $k+1$ candidate colocations from size $k$ prevalent colocations.

The apriori-gen function takes as argument $P_{k}$, the set of all prevalent size $k$ colocations. The function works as follows: First, in the join step, we join $P_{k}$ with $P_{k}$. This step is specified in a SQL-like syntax as follows:

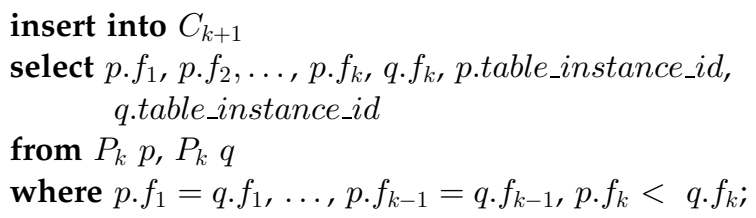

Next, in the prune step, we delete all colocations $c \in C_{k}$ such that some size $k-1$ subset of $c$ is not in $P_{k}$ :

forall colocation $c \in C_{k+1}$ do

forall size $k-1$ subsets $s$ of $c$ do if $\left(s \notin P_{k}\right)$, then delete $c$ from $C_{k+1}$;

Note that the column $f_{i}$ of $P_{k}$ refers to the $i$ feature of colocations in table $P_{k}$ and the column table_instance_id of table $P_{k}$ refers to table instances of appropriate colocations.

\subsection{Generation of Table Instances of Candidate Colocations}

Computation for generating size $k+1$ candidate colocations can be expressed as the following join query: forall colocation $c \in C_{k+1}$

insert into $T_{c} /{ }^{*} T_{c}$ is the table instance of colocation $c^{*} /$

select $p$.instance $1, p$.instance $2, \ldots, p$. instance $_{k}$, $q$.instance ${ }_{k}$

from $c$.table_instance_id ${ }_{1} p$, c.table_instance_id $2 q$

where $p$.instance ${ }_{1}=q$. instance $_{1}, \ldots$, $p \cdot$ instance $_{k-1}=q \cdot$ instance $_{k-1},\left(p\right.$. instance $_{k}$, $q$.instance $\left.{ }_{k}\right) \in \mathcal{R}$;

end;

The query takes the size $k+1$ candidate colocation set $C_{k+1}$ and table instances of the size $k$ prevalent colocations as arguments and works as follows: c.table_instance $i d_{1}$ and c.table_instance $i d_{2}$ specify the table instances of the two colocations joined in apriori_gen to produce $c$. Here, a sortmerge join is preferred because the table instances of each iteration can be kept sorted for the next iteration. This follows from a similar property of apriori-gen [2]. Sort order is based on an ordering of the set of feature types to order feature types in a colocation to form the sort-field. Finally, all colocations with empty table instances will be eliminated from $C_{k+1}$.

The join computation for generating table instances has two constraints, a spatial neighbor relationship constraint

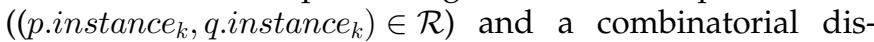
tinct event-type constraint $\left(\right.$ p.instance ${ }_{1}=$ q.instance $_{1}, \ldots$, p.instance $_{k-1}=q$. instance $\left._{k-1}\right)$. We examine three strategies for computing this join: a geometric strategy, a 
combinatorial strategy, and a hybrid strategy. These are described in forthcoming sections. Exploration of other join strategies is beyond the scope of this paper but we may explore such strategies in future work.

Geometric Approach. The geometric approach can be implemented by neighborhood relationship-based spatial joins of table instances of prevalent colocations of size $k$ with table instance sets of prevalent colocations of size 1. In practice, spatial join operations are divided into a filter step and a refinement step [18] to efficiently process complex spatial data types such as point collections in a row instance. In the filter step, the spatial objects are represented by simpler approximations such as the MBR-Minimum Bounding Rectangle. There are several well-known algorithms, such as plane sweep [3], space partition [11], and tree matching [13], which can then be used for computing the spatial join of MBRs using the overlap relationship; the answers from this test form the candidate solution set. In the refinement step, the exact geometry of each element from the candidate set and the exact spatial predicates are examined along with the combinatorial predicate to obtain the final result.

Combinatorial Approach. The combinatorial join predicate (i.e., p.instance ${ }_{1}=q$. instance $_{1}$, p. instance $_{2}=q$. instance $_{2}$, $\ldots$, p.instance $_{k-1}=$ q.instance $_{k-1}$ ) can be processed efficiently using a sort-merge join strategy [10] since the set of feature types is ordered and tables c.table_instance_id $d_{1}$ and c.table_instance_id $d_{2}$ are sorted. The resulting tuples are checked for the spatial condition ((p.instance ${ }_{k}$, q.instance $\left._{k}\right)$ $\in \mathcal{R}$ ) to get the row-instance in the result. In Fig. 2, table 4 of colocation $\{A, B\}$ and table 5 of colocation $\{A, C\}$ are joined to produce the table instance of colocation $\{A, B, C\}$ because colocation $\{A, B\}$ and colocation $\{A, C\}$ were joined in apriorigen to produce colocation $\{A, B, C\}$ in the previous step. In the example, row instance $\{3,4\}$ of table 4 and row instance $\{3,1\}$ of table 5 are joined to generate row instance $\{3,4,1\}$ of colocation $\{A, B, C\}$ (table 7 ). Row instance $\{1,1\}$ of table 4 and row instance $\{1,2\}$ of table 5 fail to generate row instance $\{1,1,2\}$ of colocation $\{A, B, C\}$ because instance 1 of $B$ and instance 2 of $C$ are not neighbors.

Hybrid Approach. The hybrid approach chooses the more promising of the spatial and combinatorial approaches in each iteration. In our experiment, it picks the spatial approach to generate table instances for colocation patterns of size 2 and the combinatorial approach for generating table instances for colocation patterns of size 3 or more.

\subsection{Pruning}

Candidate colocations can be pruned using the given threshold $\theta$ on the prevalence measure. In addition, multiresolution pruning can be used for spatial data set with strong autocorrelation [6], i.e., where instances tend to be located near each other.

Prevalence-Based Pruning. We first calculate the participation indexes for all candidate colocations in $T_{k+1}$. Computation of the participation indexes can be accomplished by keeping a bitmap of size cardinality $\left(f_{i}\right)$ for each feature $f_{i}$ of colocation $c$. One scan of the table instance of $c$ will be enough to put $1 \mathrm{~s}$ in the corresponding bits in each bitmap. By summarizing the total number of $1 \mathrm{~s}\left(p_{f_{i}}\right)$ in each bitmap, we obtain the participation ratio of each feature $f_{i}$ (divide $p_{f_{i}}$ by $\mid$ instance of $f_{i} \mid$ ). In Fig. 2 c, to calculate the participation index for colocation $\{A, B\}$, we need to calculate the participation ratios for $A$ and $B$ in colocation
$\{A, B\}$. Bitmap $b_{A}=(0,0,0,0)$ of size four for $A$ and bitmap $b_{B}=(0,0,0,0,0)$ of size 5 for $B$ are initialized to zeros. Scanning of table 4 will result in $b_{A}=(1,1,1,0)$ and $b_{B}=(1,0,0,1,0)$. Three out of four instances of $A$ (i.e., 1 , 2 , and 3) participate in colocation $\{A, B\}$, so the participation ratio for $A$ is .75. Similarly, the participation ratio for $B$ is .4. Therefore, the participation index is $\min \{.75, .4\}=.4$.

After the participation indexes are determined, prevalence-based pruning is carried out and nonprevalent colocations are deleted from the candidate prevalent colocation sets. For each remaining prevalent colocation $c$ after prevalence-based pruning, we keep a counter to specify the cardinality of the table instance of $c$. All the table instances of the prevalent colocations in this iteration will be kept for generation of the prevalent colocations of size $k+2$ and discarded after the next iteration.

Multiresolution Pruning. Multiresolution pruning is learned on a summary of spatial data at a coarse resolution using a disjoint partitioning, e.g., pagination imposed by leaves of a spatial index or a grid. A new neighbor relationship $\mathcal{R}^{c}$ on partitions is derived from relationship $\mathcal{R}$ so that two partitions are $\mathcal{R}$ neighbors if any two instances from each of the two partitions are $\mathcal{R}$ neighbors. We combine all instances of a spatial feature $f$ in each partition $s$ in the partitioning as a new coarse instance $\langle s, f, m\rangle$ in the coarse space, where $m$ is the number of instances of spatial point feature $f$ in cell $s$. For each candidate colocation generated by apriori_gen, we generate its coarse table instance using new coarse instances, new neighbor relationship $\mathcal{R}^{c}$, and its coarse participation index based on the coarse table instance. Multiresolution pruning eliminates a colocation if its coarse participation indexes fall below the threshold, because coarse participation never underestimates the participation index, as shown in Section 4.2.

We now illustrate multiresolution pruning by using a simple recti-linear grid for simplicity. In Fig. 4a, different shapes represent different point spatial feature types. Every instance has a unique ID in its spatial feature type and is labeled below it in the figure. Two instances are defined as neighbors if they are in a common $d \times d$ square. A grid with uniform cell size $d$ is superimposed on the data set. Cells $(i, j)$ refer to cells with an x-axis index of $i$ and a y-axis index of $j$. In this grid, two cells are coarse-neighbors if their centers are in a common square of size $d \times d$, which imposes an 8-neighborhood (North, South, East, West, North East, North West, South East, South West) on the cells. For example, cell-pairs $\{(0,3),(0,4)),((0,3),(1,3)\}$ and $\{(0,4),(1,3)\}$ illustrate coarse-neighbors. This coarseneighborhood definition guarantees that two cells are neighbors if there exists a pair of points from each of the two cells which are neighbors in the original data set. The process of multiresolution pruning is shown as follows.

First, we generate coarse table instances of candidate colocations of size $k+1$ by joining the coarse table instances with the coarse-neighbor relationships.

Next, we calculate the participation indexes for all candidate colocations based on the coarse table instances. For each spatial feature $f_{i}$, we add up all the counts of point instances in each coarse instance with $1 \mathrm{~s}$ in its corresponding bitmap $\left(p_{f_{i}}\right)$ and divide this by $\mid$ instance of $f_{i} \mid$ to get the coarse-participation ratio of feature $f_{i}$. For example, in Fig. $4 a$, coarse $\operatorname{pr}((\star, \circ), \star)=4 / 7$ since there are two coarse row instances of $\{\star, \circ\}$, each containing two fine-grain instances of 


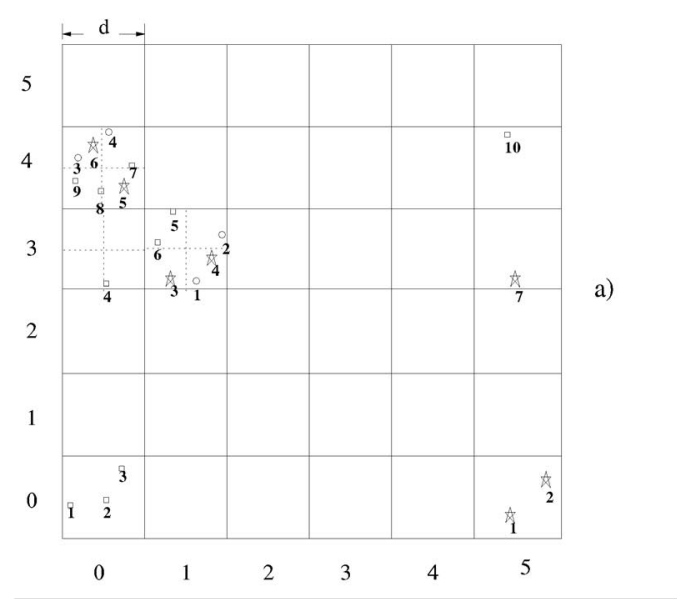

\begin{tabular}{|c|c|c|c|c|c|}
\hline \multicolumn{3}{|c|}{$\begin{array}{c}\text { Co-location of size } 3 \\
\text { and fine level table instances } \\
17\end{array}$} & \multirow{2}{*}{\multicolumn{3}{|c|}{$\begin{array}{c}\text { Co-location of size } 3 \\
\text { and coarse level table instances } \\
\text { c7 }\end{array}$}} \\
\hline \multirow{2}{*}{ 太 } & ם & $\circ$ & & & \\
\hline & 5 & 1 & $\star$ & 口 & $\circ$ \\
\hline 3 & 5 & 2 & $(0,4)$ & $(0,3)$ & $(0,4)$ \\
\hline 3 & 6 & 1 & $(0,4)$ & $(0,3)$ & $(1,3)$ \\
\hline 3 & 6 & 2 & $(0,4)$ & $(0,4)$ & $(0,4)$ \\
\hline 4 & 5 & 1 & $(0,4)$ & $(0,4)$ & $(1,3)$ \\
\hline \multirow[t]{2}{*}{4} & 5 & 2 & $(0,4)$ & $(1,3)$ & $(0,4)$ \\
\hline & ....... & & $(0,4)$ & $(1,3)$ & $(1,3)$ \\
\hline 6 & 9 & 3 & $(1,3)$ & $(0,4)$ & $(0,4)$ \\
\hline 6 & 9 & 4 & $(1,3)$ & $(0,4)$ & $(1,3)$ \\
\hline \multirow[t]{3}{*}{$4 / 7$} & $5 / 10$ & $4 / 4$ & $(1,3)$ & $(1,3)$ & $(0,4)$ \\
\hline & \multirow{2}{*}{\multicolumn{2}{|c|}{$\begin{array}{l}\text { will be pruned if min_pre } \\
\text { if set to greater than } 4 / 7 \\
\text { and algorithm stops }\end{array}$}} & $(1,3)$ & $(1,3)$ & $(1,3)$ \\
\hline & & & $-4 / 7$ & $6 / 10$ & $4 / 4$ \\
\hline
\end{tabular}

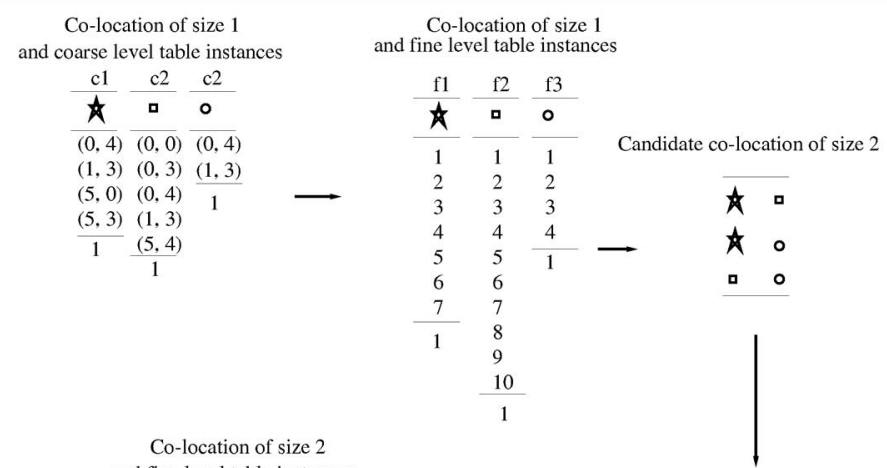

and fine level table instances

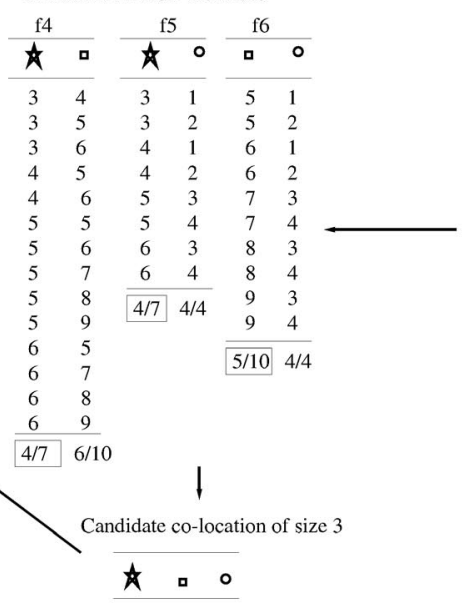

Co-location of size 2 and coarse level table instances $\stackrel{c 4}{\star} \quad \frac{c 5}{\star} \circ \frac{c 6}{\square \quad 0}$

$(0,4)(0,3)(0,4)(0,4)(0,3)(0,4)$ $(0,4)(0,4)(0,4)(1,3)(0,3)(1,3)$ $(0,4)(1,3)(1,3)(0,4) \quad(0,4)(0,4)$ $(1,3)(0,3)(1,3)(1,3)(0,4)(1,3)$ $( 1 , 3 ) ( 0 , 4 ) \longdiv { 4 / 7 } 4 / 4 \quad ( 1 , 3 ) ( 0 , 4 )$ $\begin{array}{llll}(1,3)(1,3) & 4 / 7 & 4 / 4 & (1,3)(0,4) \\ & & (1,3)(0,4)\end{array}$ $(5,3)(5,4)$ \begin{tabular}{ll|ll}
\hline $5 / 7$ & $6 / 10$ & $4 / 4$ \\
\hline
\end{tabular}

will be pruned if min_pre if set to 0.6 , $\star$ and will be further evaluated and algorithm stops

Fig. 4. Colocation miner algorithm with multiresolution pruning illustration.

$\star$ and a total of seven fine-grain instances of $\star$. Similarly, coarse $\operatorname{pr}(\{\star, \circ\}, \circ)=4 / 4=1$, yielding coarse participation index $\operatorname{pi}(\{\star, \circ\})=\min (4 / 7,4 / 4)=4 / 7$. Fig. $4 \mathrm{~b}$ shows coarse table instances of colocations $\{*, \square\},\{*, \circ\}$, and $\{\square, \circ\}$. If the threshold for prevalence is set to 0.6 , then colocation $c_{5}$ and $c_{6}$ can be pruned by multiresolution pruning. We also note that the sizes of coarse table instances are smaller than the sizes of table instances at fine resolution. This shows the possibility of computation cost saving via multiresolution pruning for clustered data sets. Finally, the examples in Fig. $4 \mathrm{~b}$ show that the coarse participation ratios and participation indexes never underestimate the true participation indexes of the original data set.

\subsection{Generating Colocation Rules}

The generate_colocation_rule function generates all the colocation rules with the user defined conditional probability threshold $\alpha$ from the prevalent colocations and their table instances. The conditional probability of a colocation rule $c_{1} \Rightarrow c_{2}$ in the event centric model is the probability of $c_{1}$ reachable to a $\mathcal{R}$-proximity neighborhood containing all the features in $c_{2}$. It can be calculated as:

$$
\frac{\mid \pi_{c_{1}}\left(\text { table_instance }\left(c_{1} \bigcup c_{2}\right)\right) \mid}{\mid \text { table_instance }\left(c_{1}\right) \mid},
$$

where $\pi$ is a projection operation with duplication elimination. Bitmaps or other data structures can be used for efficient computation using the same strategies for prevalence-based pruning.

\section{Analysis of the Colocation Mining ALGORITHMS}

Here, we analyze the colocation mining algorithms in the areas of statistical interpretation of colocation patterns, completeness, correctness, and computational complexity.

\subsection{Statistical Interpretation of the Colocation Patterns}

In spatial statistics [6], interest measures such as the cross- $K$ function, a generalization of Ripley's $K$-function [16], [17] (and variations such as the $L$-function and $G$ function) are used to identify colocated spatial feature types. The cross- $K$ function $K(h)$ for binary spatial features is defined as follows:

$$
\begin{array}{r}
K_{i j}(h)=\lambda_{j}^{-1} E[\text { number of type } j \text { instances within distance } \\
h \text { of a randomly chosen type } i \text { instance }]
\end{array}
$$

where $\lambda_{j}$ is the density (number per unit area) of type $j$ instances and $h$ is the distance. Without edge effects [7], the cross- $K$-function could be estimated by: $\hat{K}_{i j}(h)=\frac{1}{\lambda_{i} \lambda_{j} W} \sum_{k}$ $\sum_{l} I_{h}\left(d\left(i_{k}, j_{l}\right)\right)$, where $d\left(i_{k}, j_{l}\right)$ is the distance between the $k$ th instance of type $i$ and the $l$ th instance of type $j, I_{h}$ is the indicator function assuming value 1 if the distance between instance $i_{k}$ and $j_{l} d\left(i_{k}, j_{l}\right) \leq h$, and value 0 otherwise, and $W$ is the area of the study region. $\lambda_{j} \times \hat{K}_{i j}(h)$ estimates the expected number of type $j$ instances within distance $h$ of a type $i$ instances. The variance of the cross- $K$ function can be estimated by Monte Carlo simulation [6], in general, and by 


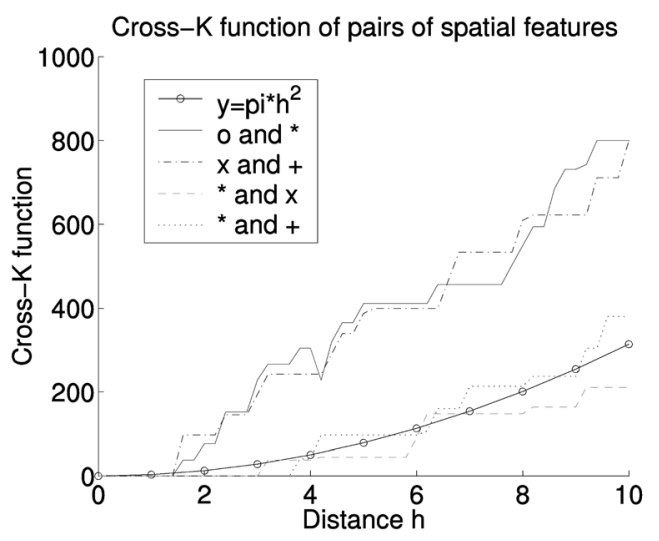

Fig. 5. Ripley’s Cross-K Function.

a close form equation under special circumstances [6]. In Fig. 5, the cross- $K$ functions of the two pairs of spatial features, i.e., $\left\{{ }^{\prime}+,^{\prime} ' x^{\prime}\right\}$ and $\left\{{ }^{\prime} \mathrm{o},{ }^{\prime}{ }^{\prime *}\right\}$, are well above the spatial complete randomness curve $y=\pi * h^{2}$, while the cross- $K$ functions of the other random two pairs of spatial features, i.e., $\left\{{ }^{*},{ }^{\prime} x^{\prime}\right\}$ and $\left\{{ }^{*},{ }^{\prime} '{ }^{\prime}\right\}$, are very close to complete spatial randomness. This figure does not show the confidence band.

We compare the participation index with the cross- $K$ function in an attempt to provide an independent method for evaluating colocation patterns. In particular, we explore the correctness of colocations using the following characterization of the relationship between the cross- $K$ function and colocations.

Lemma 1. Participation index pi $(\{A, B\})$ for colocation $\{A, B\}$ is an upper-bound on $\frac{\hat{K_{A B}}(h)}{W}$, where $\hat{K_{A B}}(h)$ is the estimation of the cross- $K$ function of colocation $\{A, B\}$ for a proximity neighborhood defined by distance $\leq h$, and $W$ is the total area of the region.

Proof. From the definition of the participation index and the definition of cross- $K$ function, we have

$$
\begin{aligned}
& \operatorname{pi}(\{A, B\})= \\
& \min \left\{\frac{\mid \pi_{A}(\text { table_instance }(A, B)) \mid}{|A|}, \frac{\mid \pi_{B}(\text { table_instance }(A, B)) \mid}{|B|}\right\}
\end{aligned}
$$

and

$$
\begin{aligned}
\frac{\hat{K_{A B}}(h)}{W} & =\frac{1}{W} \cdot \frac{1}{\lambda_{A} \lambda_{B} W} \sum_{k} \sum_{l} I_{h}\left(d\left(A_{k}, B_{l}\right)\right) \\
& =\frac{\mid \text { table_instance }(A, B) \mid}{|A| \times|B|} .
\end{aligned}
$$

We need to show only that

$$
\frac{\mid \pi_{A}(\text { table_instance }(A, B)) \mid}{|A|} \geq \frac{\mid \text { table_instance }(A, B) \mid}{|A| \times|B|}
$$

and

$$
\frac{\mid \pi_{B}(\text { table_instance }(A, B)) \mid}{|B|} \geq \frac{\mid \text { table_instance }(A, B) \mid}{|A| \times|B|} .
$$

To prove the first inequality, we need to show only that $\mid \pi_{A}($ table_instance $(\{A, B\})) \mid \geq \frac{\mid \text { table_instance }(\{A, B\}) \mid}{|B|}$. This is obvious because the total number of instances of $A$ with at least one instance of type $B$ nearby (left side) is always greater or equal to the average number of instances of type $A$ around an instance of type $B$ (right side). The second inequality could be proved in a similar manner. $\square$

Lemma 2. The table instance table_instance $(\{A, B\})$ of a binary colocation $\{A, B\}$ has enough information to compute the estimator $\frac{K_{A B}(h)}{W}$ of the cross- $K$ function for $h=d$, where distance $d$ defines the proximity neighborhood.

Proof. Since

$$
\begin{aligned}
\hat{\frac{K_{A B}(h)}{W}} & =\frac{1}{W} \cdot \frac{1}{\lambda_{A} \lambda_{B} W} \sum_{k} \sum_{l} I_{h}\left(d\left(A_{k}, B_{l}\right)\right) \\
& =\frac{\mid \text { table_instance }(A, B) \mid}{|A| \times|B|},
\end{aligned}
$$

this lemma holds.

Lemma 1 may be used to establish the correctness of colocation rules with respect to the threshold defined by $\frac{\hat{K}_{A B}(h)}{W}$, and Lemma 2 may be used to establish the colocation miner as an algorithm to efficiently compute $\hat{K}_{A B}$ for selected colocations, particularly when the multiresolution filter is effective. We are at present aware of only the use of the cross- $K$ function to characterize pairwise spatial interactions. We plan to explore spatial statistics research literature to look for measures of spatial interaction among more than two features and compare those measures to the participation index.

\subsection{Completeness and Correctness}

Lemma 3: Antimonotone. The participation ratio and participation index are antimonotone (monotonically nonincreasing) as the size of the colocation increases.

Proof. The participation ratio is antimonotonic because a spatial feature instance that participates in a row instance of a colocation $c$ also participates in a row instance of a colocation $c^{\prime}$, where $c^{\prime} \subseteq c$. The participation index is also antimonotonic because 1) the participation ratio is antimonotonic and 2)

$$
\begin{aligned}
p i\left(c \bigcup f_{k+1}\right) & =\min _{i=1}^{k+1}\left\{\operatorname{pr}\left(c \bigcup f_{k+1}, f_{i}\right)\right\} \\
& \leq \min _{i=1}^{k}\left\{\operatorname{pr}\left(c \bigcup f_{k+1}, f_{i}\right)\right\} \\
& \leq \min _{i=1}^{k}\left\{\operatorname{pr}\left(c, f_{i}\right)\right\}=p i(c) .
\end{aligned}
$$

Lemma 4. The coarse participation index computed by multiresolution pruning never underestimates the participation indexes of the original data set. The candidate colocation set found is a superset of the prevalent colocation set on the original data set.

Proof. When colocation size $=1$, the value of the coarse participation index and the true participation index is 1 , so Lemma 4 is trivially true. Suppose Lemma 4 is true for colocations size $=k$. Let us consider the case that 
colocation size is equal to $k+1$. For each candidate colocation $c$ of size $k+1$ generated from the apriori_gen by joining $c_{1}$ and $c_{2}$ of size $k$, we generate its coarse instance table by joining the coarse instance tables of $c_{1}$ and $c_{2}$. Because Lemma 4 is true for colocations of size $k$, the candidate colocation set of size $k$ found is a superset of the prevalent colocation set on the original data set. Thus, $c_{1}$ and $c_{2}$ are in the candidate colocation set in the previous iteration and their coarse level table instances are available to be joined to produce the coarse level table instance of $c$. The table join to produce the coarse table instance of $c$ has the following property: If $\mathcal{R}\left(p_{1}, p_{2}\right)$ is in the original data set, then coarse $\mathcal{R}^{c}\left(\right.$ cell $c_{1}$, cell $\left.c_{2}\right)$ will be in the coarse-level data set given $p_{1} \in c_{1}$ and $p_{2} \in c_{2}$. When we calculate the coarse participation index, any spatial feature instance which participates in the colocation in the original data set will contribute to the counts during the coarse participation ratio calculation. So, the coarse participation ratios never underestimate the true participation ratios, implying that the coarse participation index never underestimates the true participation index and that the pruning will not eliminate any truly prevalent colocation. Thus, the candidate colocation set after multiresolution pruning is a superset of the prevalent colocation set on the original data set.

Lemma 5: Completeness. The Colocation Miner algorithm is complete.

Proof. The schema level pruning using apriori_gen is complete due to the monotonicity of the participation index as proved in Lemma 3. Then, we prove that the join of the table instances of $c_{1}$ and $c_{2}$ to produce the table instance of $c$ is complete. According to the proximity neighborhood definition, any subset of a proximity neighborhood is a proximity neighborhood too. For any instance $I=\left\{i_{1}, \ldots, i_{k+1}\right\}$ of colocation $c$, subsets $I_{1}=$ $\left\{i_{1}, \ldots, i_{k}\right\}$ and $I_{2}=\left\{i_{1}, \ldots, i_{k-1}, i_{k+1}\right\}$ are neighborhoods, $i_{k}$ and $i_{k+1}$ are neighbors, and $I_{1}$ and $I_{2}$ are row instances of $C_{1}$ and $C_{2}$, respectively. Joining $I_{1}$ and $I_{2}$ will produce $I$. Enumeration of the subsets of each of the prevalent colocations ensures that no spatial colocation rules with both high prevalence and high conditional probabilities are missed. We then prove that multiresolution pruning does not affect completeness. By Lemma 4, the colocation set found is a superset of the prevalent colocation set on the original data set. Thus, multiresolution pruning does not falsely eliminate any prevalent colocation.

\section{Lemma 6: Correctness. The Colocation Miner is correct.}

Proof. We will show only that the row instance of each colocation is correct, as that will imply the correctness of the participation index values and that of each colocation meeting the user specified threshold. An instance $I_{1}=$ $\left\{i_{1,1}, \ldots, i_{1, k}\right\}$ of $c_{1}=\left\{f_{1}, \ldots, f_{k+1}\right\}$ and an instance $I_{2}=$ $\left\{i_{2,1}, \ldots, i_{2, k}\right\}$ of $c_{2}=\left\{f_{1}, \ldots, f_{k-1}, f_{k+1}\right\}$ is joined to produce an instance $I_{\text {new }}=\left\{i_{1,1}, \ldots, i_{1, k}, i_{2, k}\right\}$ of $c=\left\{f_{1}, \ldots\right.$, $\left.f_{k+1}\right\}$ if: 1) all elements of $I_{1}$ and $I_{2}$ are the same except $i_{1, k}$ and $\left.i_{2, k} ; 2\right) i_{1, k}$ and $i_{2, k}$ are neighbors. The schema of $I_{\text {new }}$ is apparently $c$, and elements in $I_{\text {new }}$ are in a proximity neighborhood because $I_{1}$ is a proximity neighborhood and $i_{2, k}$ is a neighbor of every element of $I_{1}$.

\subsection{Computational Complexity Analysis}

This section examines the strategies for generating candidate colocations, the evaluation of the multiresolution pruning strategy, and the effect of noise. First, there are two basic strategies for generating table instances of candidate colocations, namely, the geometric approach and the combinatorial approach. For generating size-2 colocations, the combinatorial approach ends up being the nest-loop join strategy with an asymptotic complexity of $O\left(N^{2}\right)$, while the geometric approach has the CPU $\operatorname{cost}^{1}$ of $O(N \log N+M)$, where $N$ is the total number of instances of all features and $\mathrm{M}$ is the number os intersections. When the data set is sparse, the cost of the combinatorial approach will be much higher. However, when generating table instances of colocations of size 3 or more, the combinatorial approach becomes cheaper than the geometric approach. This is due to its exploitation of the sort-merge join strategy while keeping each table instance sorted. In a hybrid approach, we pick the cheaper of the two basic strategies in each iteration to achieve the best overall cost.

Second, let us compare the cost of the Colocation Miner algorithm with and without the multiresolution pruning step. Let $T_{m c m}(k)$ and $T_{c m}(k)$ represent the costs of iteration $k$ of the Colocation Miner algorithm with and without the multiresolution pruning.

$$
\begin{aligned}
T_{m c m}(k)= & T_{\text {apriori_gen }(C(\text { prev }, k))}+T_{\text {prune }(C(\text { cand }, k+1), \text { griddata })} \\
& +T_{\text {prune }(C(\text { sub_cand }, k+1), \text { data })} \\
T_{\text {cm }}(k)= & T_{\text {apriori_gen }(C(\text { prev }, k))}+T_{\text {prune }(C(\text { cand }, k+1), \text { data }) .} .
\end{aligned}
$$

In (1), $T_{\text {apriori_gen }(C(p r e v, k))}$ represents the cost of apriori_gen based on the prevalent colocation set of size k. Here, resolution is not relevant since apriori_gen works on the spatial feature level only. $T_{\text {prune }(C(c a n d, k+1) \text {,grid data })}$ represents the cost for multiresolution pruning on the coarse level data set in iteration k. After coarse-level pruning, we only need to search the leftover subset of the original data set. $T_{\text {prune(C(sub_cand, } k+1), d a t a)}$ represents the cost for fine level instance pruning on the leftover subsets of the original data set. In addition, $T_{\text {prune }(C(\operatorname{cand}, k+1), \text { data })}$ represents the cost for fine level instance pruning on the original data set in iteration $\mathrm{k}$.

The bulk of time is consumed in generating table instances and calculating the participation indexes; thus, the ratio can be simplified as:

$$
\frac{T_{\text {mcm }}(k)}{T_{\text {cm }}(k)} \approx \frac{T_{\text {prune }(C(\text { cand }, k+1), \text { griddata })}+T_{\text {prune }(C(\text { sub_cand }, k+1), \text { data })}}{T_{\text {prune }(C(\text { cand }, k+1), \text { data })}} .
$$

Furthermore, we assume that the average time to generate a table instance in the original data set is $T_{\text {orig }}(k)$ for iteration $\mathrm{k}$ and the average time to generate a table instance in the grid data set is $T_{\text {grid }}(k)$ for iteration $k$. The number of candidate colocations generated by the apriori_gen is $\left|C_{k+1}\right|$ and the number of candidate colocations after the coarse instance level pruning is $\left|C_{k+1}^{\prime}\right|,(2)$ can be written as:

1. The $\mathrm{I} / \mathrm{O}$ costs of the geometric approach and the combinatorial approach are similar. 


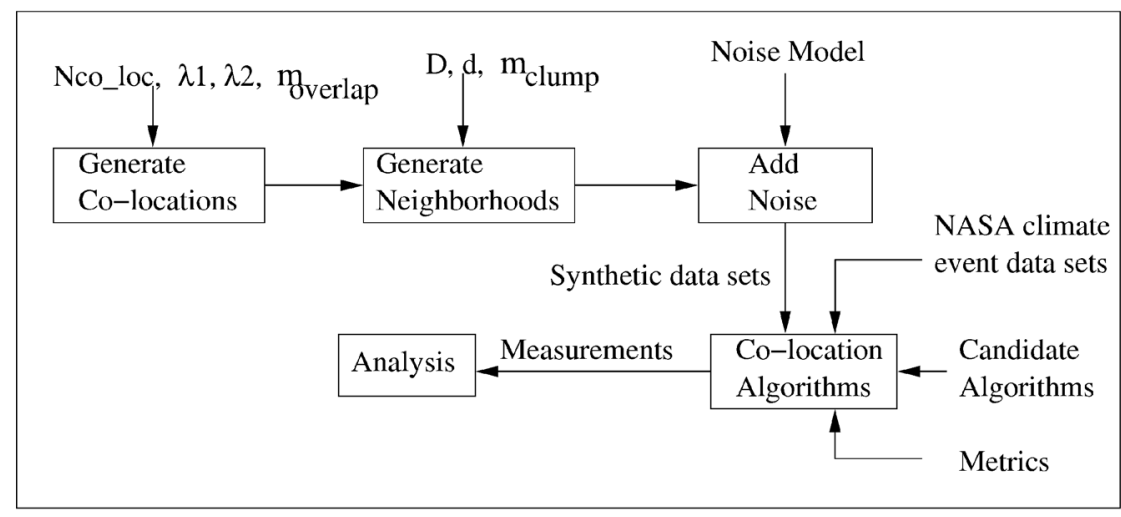

Fig. 6. Experimental setup and design.

$$
\begin{aligned}
\frac{T_{\text {mcm }}(k)}{T_{\text {cm }}(k)} & \approx \frac{\left|C_{k+1}\right| \times T_{\text {grid }}(k)+\left|C_{k+1}^{\prime}\right| \times T_{\text {orig }}(k)}{\left|C_{k+1}\right| \times T_{\text {orig }}(k)} \\
& =\frac{T_{\text {grid }}(k)}{T_{\text {orig }}(k)}+\frac{\left|C_{k+1}^{\prime}\right|}{\left|C_{k+1}\right|} .
\end{aligned}
$$

The first term of the ratio is controlled by the "clumpiness" (the average number of instances of the spatial features per grid cell) of the locations of spatial features. The second term is controlled by the filtering efficiency of the coarse instance level pruning. When the locations of spatial features are clustered, the sizes of the fine level table instances are much greater than the sizes of the coarse level table instances and the time needed to generate fine level table instances is greater than the time needed to generate coarse level table instances. In our experiments, as described in the next section, we use the parameter $m_{\text {clump }}$, which controls the number of instances clumping together for each spatial feature, to evaluate the first term, and we use the parameter $m_{\text {overlap, }}$ which represents the possible false candidate ratios, to evaluate the second term. From the formula, we can see that the colocation miner with multiresolution pruning is likely to be more efficient than the colocation miner without multiresolution pruning when the locations of spatial features are clustered and the false candidate ratio is high.

\section{Experimental Performance Evaluation}

Fig. 6 describes the experimental setup to evaluate the impact of design decisions on the relative performance of the colocation miner algorithm. We evaluated the performance of the algorithms with synthetic and real-world NASA climate data sets. Synthetic data sets are generated using a methodology similar to methodologies used to evaluate algorithms for mining association rules [2]. Synthetic data sets allow better control towards studying the effects of interesting parameters.

A data-flow diagram of the data generation process is shown in Fig. 6. The process began with the generation of core colocation subsets of spatial features. To generate a subset of features, we first chose the size of the subset from a Poisson distribution with mean $\left(\lambda_{1}\right)$. Then, a set of features for this core colocation pattern was randomly chosen. For each core colocation, $m_{\text {overlap }}$ maximal colocations were generated by appending one more spatial feature to a core colocation. The larger $m_{\text {overlap }}$ is, the more false candidate apriori_gen generates. The size of each table instance of each colocation was chosen from another Poisson distribution with mean $\lambda_{2}$. Next, we generated the set of proximity neighborhoods for colocations instances using the size of their table instances from the previous step. $m_{\text {clump }}$ point locations for each feature in the colocation were embedded inside a proximity neighborhood of size $d$. The locations of proximity neighborhoods were chosen at random in the overall spatial framework. For simplicity, the shape of the overall spatial framework was a rectangle of size $D_{1} \times D_{2}$ and the size of each proximity neighborhood was $d \times d$. The final step involved adding noise. The model for noise used two parameters, namely, the ratio of noise features $r_{\text {noise }} f$ and the number of noise instances $p_{\text {noise_n } n}$. Noise was added by generating a set of instances of features from a set of noise features disjoint with the features involving generation of core colocations and placing the instances at random locations in the global spatial framework.

The real-world NASA climate data used in our experiments contain monthly measurements of various monthly numeric climate variables, e.g., precipitation and sea surface temperature, over a period of 12 years, starting in January 1982. Events, such as drought, wet, and hot, are defined via statistical thresholding using mean and standard deviation as detailed in [20].

Our experiments were performed on a Sun Ultra 10 workstation with a $440 \mathrm{MHz}$ CPU and 128 Mbytes memory running the SunOS 5.7 operating system.

\subsection{Comparing Strategies for Generating Table Instances}

In this section, we compare the geometric, the combinatorial, and the hybrid strategies using synthetic and real-world NASA climate data sets. The synthetic data set, generated using parameter values in column C1 of Table 1 , used a rectangle spatial framework of size $10^{6} \times 10^{6}$, a square proximity neighborhood of size $10 \times 10$, an average colocation size of 5 , an average table instance size of 50 when $m_{\text {clump }}=1$, a noise feature ratio of 0.5 , a noise number of 50,000 , and an overlapping degree of 1 .

Fig. 7a shows the execution times for the three candidates with the prevalence threshold set to 0.9. In the figure, the first column reports the execution time needed to discover colocations of size 2. As can be seen, the geometric strategy is faster than the combinatorial strategy for generating size-2 colocations. Spatial-join data structures help the geometric algorithm in this step. Also, the remaining columns in Fig. 7a report the total execution 
TABLE 1

Parameters Used to Generate the Synthetic Data

\begin{tabular}{|l|l|l|l|}
\hline Parameter & Definition & C1 & C2 \\
\hline \hline$N_{\text {co_loc }}$ & The number of core co-locations & 5 & 4 \\
\hline$\lambda_{1}$ & $\begin{array}{l}\text { The parameter of the Poisson distribution to define the size of the core } \\
\text { co-locations }\end{array}$ & 5 & 5 \\
\hline$\lambda_{2}$ & $\begin{array}{l}\text { The parameter of the Poisson distribution to define the size of the table } \\
\text { instance of each co-location when } m_{\text {clump }}=1\end{array}$ & 50 & 50 \\
\hline$D_{1} \times D_{2}$ & The size of the spatial framework & $10^{6} \times$ & $250 \times$ \\
\hline$d$ & The size of the square to define a co-location & $10^{6}$ & 1,000 \\
\hline$r_{\text {noise } f}$ & $\begin{array}{l}\text { The ratio the of number of noise features over the number of features } \\
\text { involved in generating the maximal co-locations }\end{array}$ & 10 & 10 \\
\hline$r_{\text {noise_n }}$ & The number of noise instances & 5 & .5 \\
\hline$m_{\text {overlap }}$ & $\begin{array}{l}\text { The number of co-location generated by appending one more spatial } \\
\text { feature for each core co-location }\end{array}$ & 1 & 1 \\
\hline$m_{\text {clump }}$ & $\begin{array}{l}\text { The number of instances generated for each spatial feature in a } \\
\text { proximity neighborhood for a co-location }\end{array}$ & 1 & 1 \\
\hline
\end{tabular}

time to discover all the colocations as well as the time to discover colocations of size 3 or more, given prevalent colocations of size 2. In these cases, the combinatorial algorithm is orders of magnitude faster than the geometric algorithm. A sort-merge join strategy (e.g., apriori-gen [2]) helps the combinatorial algorithm. The hybrid strategy uses the geometric algorithm for discovering prevalent colocations of size 2 and the combinatorial algorithm for discovering larger colocations. Thus, it is expected to achieve the best overall performance.

Similar trends were also observed for the NASA climate event data set, as shown in Fig. $7 \mathrm{~b}$. In this experiment, the prevalence threshold is set to 0.3 and the grid size is 4 by 4 . All events are extracted at the threshold 1.5 using Z-score transformation.

\subsection{Effect of the Filter}

The effect of the multiresolution pruning filter was evaluated with spatial data sets generated using parameter values shown in column $\mathrm{C} 2$ of Table 1 . We used a

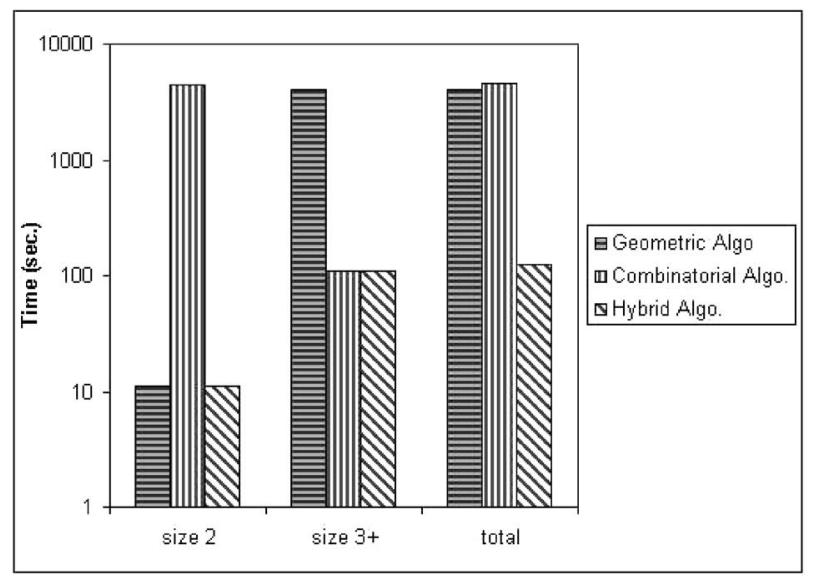

(a) rectangular spatial framework of size $250 \times 1,000$, a square proximity neighborhood of size $10 \times 10$, an average colocation size of 5 , an average table instance size of 50 when $m_{\text {clump }}=1$ a noise feature ratio of 0.5 , a noise number of 1000 , a core colocation size of 4 , and an overlapping degree of 1. Spatial framework sizes were proportional to the total number of instances to avoid unexpected patterns created by overcrowding of instances. The overlapping degree $\left(m_{\text {overlap }}\right)$ was set from 2 to 8 and the clumpiness measure $\left(m_{\text {clump }}\right)$ was set from 5 to 20 to generate other data sets. We ran the Colocation Miner with and without multiresolution pruning on these data sets. Prevalence thresholds were set to the estimation of the actual prevalences from the generation of the data sets.

Fig. 8 summarizes the performance gain by using multiresolution pruning. The $x$-axis represents the overlap degree, which controls the false candidates generated by apriori_gen in the first figure or the "clumpiness" of locations of instances of spatial feature type in the second figure. The $y$-axis represents the ratio of runtime of the

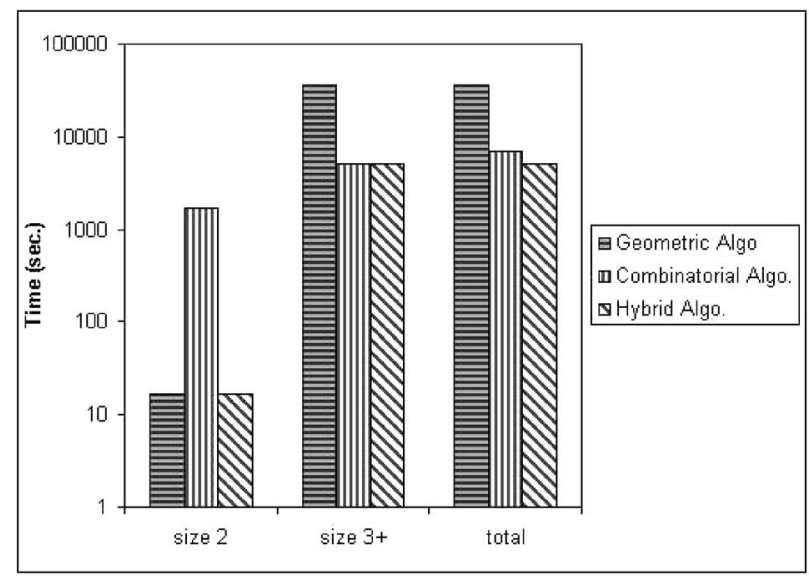

(b)

Fig. 7. (a) Relative performance of geometric, combinatorial, and hybrid algorithms on the synthetic data set. (b) Relative performance of geometric, combinatorial, and hybrid algorithms on the NASA climate data set. 


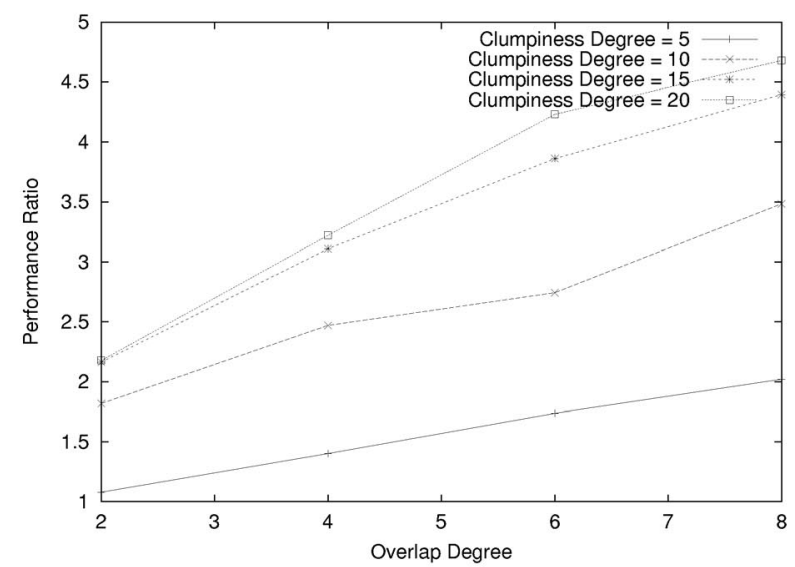

(a)

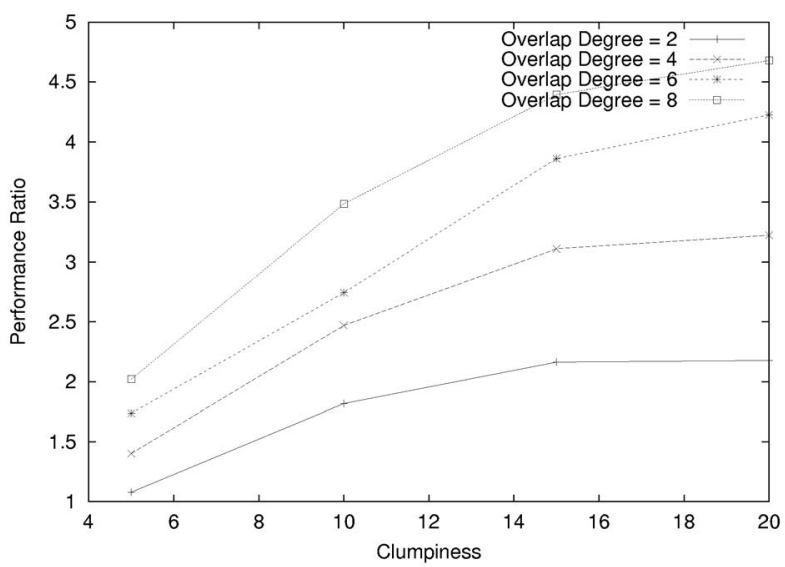

(b)

Fig. 8. Filter time ratio by (a) overlap degree and by (b) clumpiness degree,

Colocation Miner without multiresolution pruning to the runtime with multiresolution pruning. The results show that, as the degree of overlap and the number of false candidates increase, the running time is reduced by a factor of 1 to 4.5 .

Fig. 9 summarizes the ratio of the computation time for multiresolution pruning and for prevalence-based pruning. The $x$-axis represents the overlap degree or the "clumpiness" of the locations of each feature type. The overhead of multiresolution pruning as a fraction of prevalence-based pruning decreases when the degree of overlap or clumpiness increases. Clumpiness affects the overhead, reducing it from 0.45 to 0.1 .

\subsection{Effect of Noise}

The base data set, generated using parameter values in column C1 of Table 1, used a rectangle spatial framework of size $10^{6} \times 10^{6}$, a square proximity neighborhood of size $10 \times 10$, an average colocation size of 5 , an average table instance size of 50 when $m_{\text {clump }}=1$, a noise feature ratio of 0.5 , a noise number of 50,000 , and an overlapping degree of

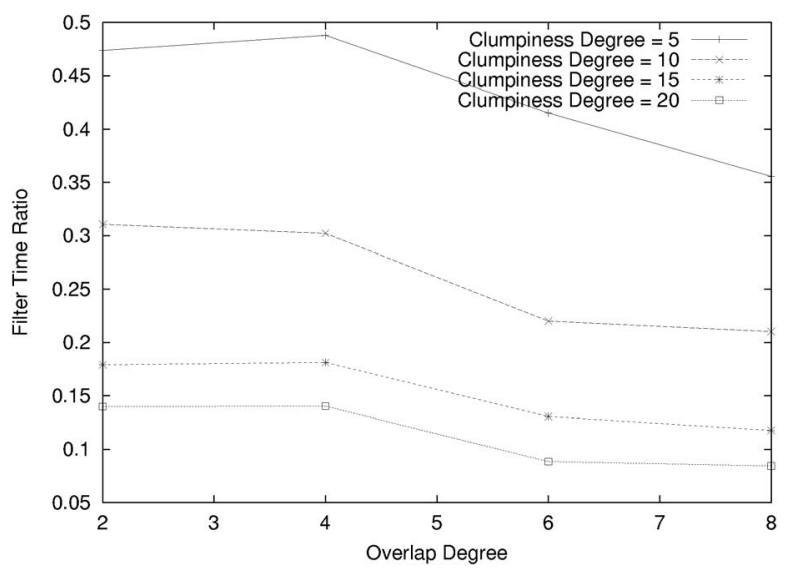

(a)
1. Then, we increased the noise instances up to 800,000 and measured the performance, as shown in Fig. 10. The execution time for discovering colocations of size 2 and $3+$ are shown in the figure. The results show that noise level affects the execution time to discover colocations of size 2, but does not affect the execution time to discover larger colocations given colocations of size 2 . In other words, noise is filtered out during the determination of colocations of size 2.

\section{Discussion}

In this section, we present a detailed comparison of our approach with the closest related work [15] for pattern semantics and algorithmic ideas.

- Pattern Semantics. Morimoto [15] defined distancebased patterns called k-neighboring class sets. In this work, the number of instances for a pattern is used as its prevalence measure. However, this measure may not possess an antimonotone property if the instances overlap; that is, the number of instances

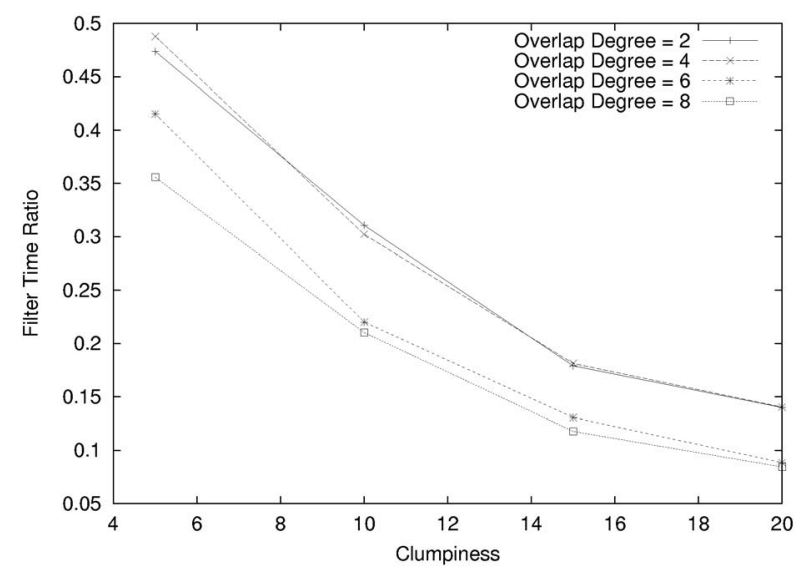

(b)

Fig. 9. Filter time ratio by (a) overlap degree and by (b) clumpiness degree. 


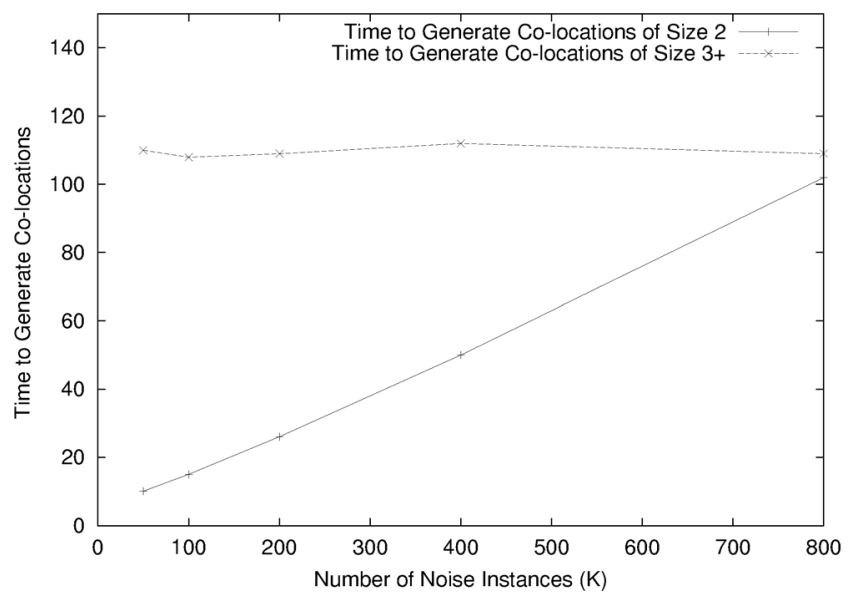

Fig. 10. Noise effect on the colocation miner.

may increase with the increase of the pattern size. For example, in Fig. 11, there are two overlapping instances: $\left\{{ }^{\prime \prime} 2, "\right.$ " 8 " $\}$ and $\{$ " 1, " " 8 " $\}$ of the set \{square, triangle\}. These two instances share one common instance $\{$ " 8 " $\}$ of the triangle feature. Indeed, there is only one instance of the triangle feature. In other words, the number of instances of the set \{square, triangle\} is greater than the number of instance of its subset \{triangle\}. To deal with this issue, Morimoto [15] used the following constraint to get the antimonotone property.

"any point object must belong to only one instance of a kneighboring class set."

As shown in Fig. 11, with this constraint, only one instance $\{2,8\}$ is specified for the 2-neighboring class set \{square, triangle\}. However, this constraint may lead to the following difficulty as described in [15].

"Instances of k-neighboring class set for k > 2 may (be) different depending on the order of the class as added into the class set. Therefore, the support value of a k-neighboring class set for $\mathrm{k}>2$ may be slightly different."

Our approach does not need the constraint of "any point object must belong to only one instance" since we do not use the number of instances for a pattern as its prevalence measure. We propose the participation index as the prevalence measure, which possesses a desirable antimonotone property. A unique subset of colocation patterns can be specified using a threshold on the participation index without consideration of algorithmic details such as the order of examination of instances of a colocation. In addition, the correctness and completeness of colocation mining algorithms can be defined using the participation index.

- Algorithmic Ideas. We have noted that a direct performance comparison of our algorithm with the algorithm by Morimoto [15] is not very meaningful due to the difference in the prevalence measure and, thus, the set of identified patterns. Nonetheless, we provide a comparison of the algorithmic ideas now. Morimoto [15] provided an iterative algorithm for mining neighboring class sets with $k+1$ features from those with $\mathrm{k}$ features. In his algorithm, a nearest neighbor based spatial join was applied in each iteration. More specifically, a geometric technique, a Voronoi diagram, was used to take advantage of the restriction that "any point object must belong to only one instance of a k-neighboring class set." This algorithm considers a pure geometric join approach. In contrast, our colocation mining algorithm considers a combinatorial join approach in addition to a pure geometric join approach to generate size $\mathrm{k}+1$ colocation patterns from size- $\mathrm{k}$ colocation patterns. Our experimental results show that a hybrid of geometric and combinatorial methods results in lower computation cost than either a pure geometric approach or pure combinatorial approach. In addition, we apply a multiresolution filter to exploit the spatial autocorrelation property of spatial data for effectively reducing the search space.

\section{Conclusion and Future Work}

In this paper, we formalized the colocation problem and showed the similarities and differences between the colocation rules problem and the classic association rules problem as well as the difficulties in using traditional measures (e.g., support, confidence) created by implicit, overlapping and potentially infinite transactions in spatial data sets. We proposed the notion of user-specified proximity neighborhoods in place of transactions to specify groups of items and defined interest measures that are

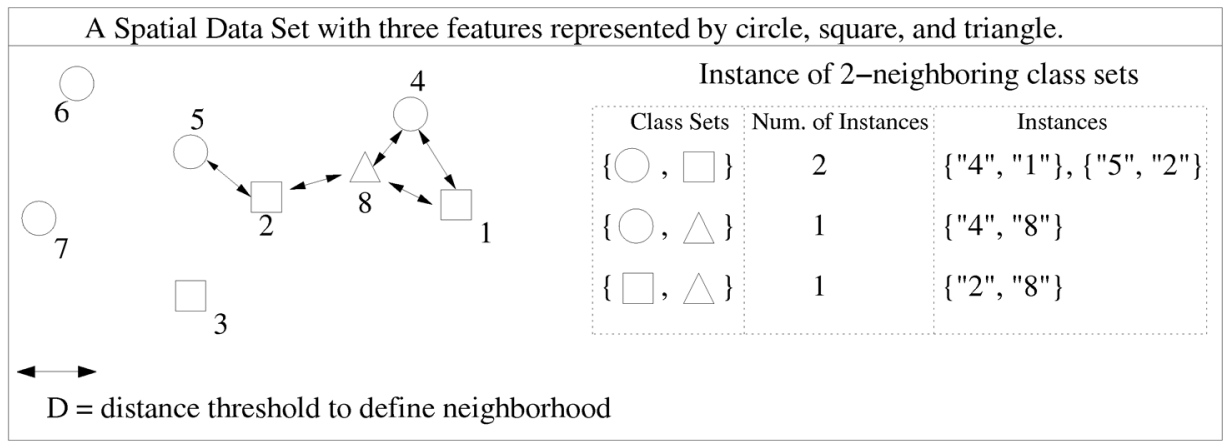

Fig. 11. A spatial data set with three features represented by circle, square, and triangle. This sample data set is from Morimoto's paper [15]. In total, there are eight instances for three features. An edge connects two instances if the distance between these two instances is less than the distance threshold. 
robust in the face of potentially infinite overlapping proximity neighborhoods. A key observation was that some properties of proximity neighborhood cliques obey the downward inclusion property necessary for a priori-based rule mining. The cardinality of table instances does not obey this property but the proposed participation index does, allowing interactive pruning. In addition, the participation index has a spatial statistical interpretation as an upperbound on the cross- $K$ function, a classical spatial statistical measure of association for binary spatial features. In contrast, related approaches [12], [15] have not provided spatial statistical interpretations of their results.

The Colocation Miner, an algorithm for mining colocation patterns, was presented and analyzed for correctness, completeness, and computation cost. Design decisions in the proposed algorithm were evaluated using theoretical and experimental methods. Empirical evaluation shows that the geometric strategy performs much better than the combinatorial strategy when generating size-2 colocations; however, it becomes slower when generating colocations with more than two features. The hybrid strategy integrates the best features of the above two approaches. Furthermore, when the locations of the features tend to be spatially clustered, which is often true for spatial data due to spatialautocorrelation, the computation cost of the colocation miner can be significantly reduced with a multiresolution filter.

Several questions remain open. First, the choice of neighbor relation $\mathcal{R}$ does impact the performance of the proposed algorithms. We plan to examine statistical methods, e.g., interinstance distance histograms, to develop guidelines for the selection of $\mathcal{R}$. Second, the colocation mining problem should be investigated to account for extended spatial data types, such as line segments and polygons. Also, we considered only Boolean features here. In the real world, the features can be categorical and continuous. There is a need to extend the colocation mining framework to handle continuous features. Third, we plan to evaluate the impact of multiresolution filtering on overall performance of the proposed algorithms using real world data sets which exhibit strong spatial autocorrelation. Finally, if locations of features change over time, it is possible for us to identify some spatiotemporal association patterns. Quantitative association, e.g., (A,A), and quantitative association rules, e.g., $(A \Rightarrow A)$, may also be explored in the future.

\section{ACKNOWLEDGMENTS}

The authors would like to thank Raymod T. Ng (University of British Columbia, Canada), Jiawei Han (Simon Fraser University, Canada), James Lesage, and Sucharita Gopal (Boston University) for valuable insights. They also would like to thank C.T. Lu, Weili $\mathrm{Wu}$, and Pusheng Zhang (University of Minnesota) for their valuable feedback. They are grateful to Kim Koffolt for her timely and detailed feedback that helped to improve the readability of this paper.

\section{REFERENCES}

[1] Open GIS Consortium, Inc., http://www.opengis.org, 2004.

[2] R. Agarwal and R. Srikant, "Fast Algorithms for Mining Association Rules," Proc. 20th Int'l Conf. Very Large Data Bases, 1994.
[3] L. Arge, O. Procopiuc, S. Ramaswamy, T. Suel, and J. Vitter, "Scalable Sweeping-Based Spatial Join," Proc. Int'l Conf.Very Large Databases, 1998

[4] C. Berge, Graphs and Hypergraphs. American Elsevier, 1976.

[5] Y. Chou, Exploring Spatial Analysis in Geographic Information System. Onward Press, 1997.

[6] N.A.C. Cressie, Statistics for Spatial Data. Wiley and Sons, 1991.

[7] P. Dixon, "Ripley's K Function," http://www.stat.iastate.edu/ preprint/articles/2001-18.pdf, Dec. 2001.

[8] V. Estivill-Castro and I. Lee, "Data Mining Techniques for Autonomous Exploration of Large Volumes of Geo-Referenced Crime Data," Proc. Sixth Int'l Conf. Geocomputation, 2001.

[9] V. Estivill-Castro and A. Murray, "Discovering Associations in Spatial Data-An Efficient Medoid Based Approach," Proc. Second Pacific-Asia Conf. Knowledge Discovery and Data Mining, 1998.

[10] G. Graefe, "Sort-Merge-Join: An Idea Whose Time has (h) Passed?" Proc. IEEE Conf. Data Eng., 1994.

[11] D. J. DeWitt and J.M. Patel, "Partition Based Spatial-Merge Join," Proc. ACM SIGMOD Conf. Management of Data, June 1996.

[12] K. Koperski and J. Han, "Discovery of Spatial Association Rules in Geographic Information Databases," Proc. Fourth Int'l Symp. Spatial Databases, 1995.

[13] S.T. Leutenegger and M.A. Lopez, "The Effect of Buffering on the Performance of R-Trees," Proc. Int'l Conf. Data Eng., 1998.

[14] S. Lipschutz, Schaum's Outline of General Topology. McGraw-Hill Trade, 1968

[15] Y. Morimoto, "Mining Frequent Neighboring Class Sets in Spatial Databases," Proc. ACM SIGKDD Int'l Conf. Knowledge Discovery and Data Mining, 2001.

[16] B.D. Ripley, "The Second-Order Analysis of Stationary Point Process," J. Applied Probability, vol. 13, pp. 255-266, 1976.

[17] B.D. Ripley, "Modelling Spatial Patterns," J. Royal Statistical Soc. Series B, vol. 39, pp. 172-192, 1977.

[18] S. Shekhar and S. Chawla, Spatial Databases: A Tour. Prentice Hall, 2003.

[19] S. Shekhar and Y. Huang, "Colocation Rules Mining: A Summary of Results," Proc. Seventh Int'l Symp. Spatio-Temporal Databases, 2001.

[20] P. Tan, M. Steinbac, V. Kumar, C. Potter, and S. Klooster, "Finding Spatio-Temporal Patterns in Earth Science Data," Proc. KDD Workshop Temporal Data Mining, 2001.

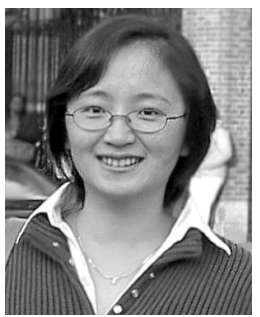

Yan Huang received the BS degree in computer science from Peking University, Beijing, China, in July 1997 and the PhD degree in computer science from University of Minnesota, TwinCities, Minnesota, in July 2003. She is currently an assistant professor in the Computer Science and Engineering Department at the University of North Texas, Denton, Texas. Her research interests include databases, spatial databases, data mining, and geographic information systems (GIS). She is a member of IEEE Computer Society and ACM SIGMOD. 


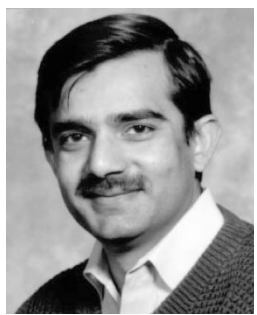

Shashi Shekhar received the BTech degree in computer science from the Indian Institute of Technology, Kanpur, India, in 1985, the MS degree in business administration, and the PhD degree in computer science from the University of California at Berkeley, in 1989. He is currently a professor of computer science at the University of Minnesota, Minneapolis. His research interests include spatial databases, spatial data mining, geographic and information systems (GIS), and intelligent transportation systems. He is a coauthor of a textbook on spatial databases (Prentice Hall, 2003) and has published more than 100 research papers in peer-reviewed journals, books, conferences, and workshops. He is a coeditor-in-chief of GeoInformatica: An International Journal on Advances of Computer Science for GIS, and has served on the editorial boards of IEEE Transactions on Knowledge and Data Engineering as well as the IEEE-CS Computer Science \& Engineering Practice Board. He also served as a program cochair of the ACM International Workshop on Advances in Geographic Information Systems, 1996. He is serving as a member of the mapping science committee of the National Research Council National Academy of Sciences (2004-2006) and has served as a member of the Board of Directors of the University Consortium on GIS (2003-2004). Dr. Shekhar is a fellow of the IEEE Computer Society and a member of the ACM.

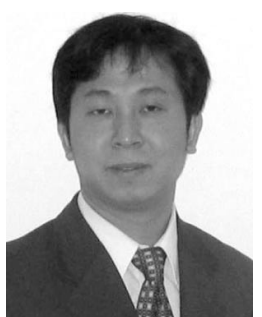

Hui Xiong received the $B E$ degree in automation from the University of Science and Technology of China, Hefei, in 1995, and the MS degree in computer science from the National University of Singapore, in 2000. He is currently a PhD candidate in the Department of Computer Science and Engineering at the University of Minnesota, Minneapolis. His research interests include data mining, spatial databases, geographic and information systems, and statistical computing. He is a student member of the IEEE Computer Society and the ACM.

$\triangleright$ For more information on this or any other computing topic, please visit our Digital Library at www.computer.org/publications/dlib. 